\title{
CHANGES IN THE IMMUNOLOGIC MARKERS OF ELASTIN DEGRADATION IN SUBJECTS WITH METABOLIC SYNDROME
}

\author{
Edward Mekenyan, Nadya Stancheva, Snejana Tisheva \\ First Cardiological Clinic UMHAT "G. Stranski" - Pleven, \\ Medical University Pleven, Bulgaria.
}

\begin{abstract}
:
Background: It is known that metabolic syndrome characterized by diabetes, hypertension, dyslipidemia, and central obesity is associated with the syndrome of early vessels aging, characterized by a change in elasticity of the vessel wall. The early manifestation of the metabolic syndrome in younger people in the modern society, leads to earlier manifestation of the complications of early vessels aging, and the combination of several risk factors is crucial and leads to acceleration of the vessels aging. Elastin is one of the main building blocks of the of the vessel wall. Its main characteristic is its elasticity, allowing the vessel to restore its shape after stretching or shrinking. Loss of elasticity is a key component in the pathogenesis of cardiovascular complications.
\end{abstract}

Materials and methods: A study is conducted on 62 subjects with metabolic syndrome without vascular complications and 42 controls. The main objective of the study was to compare the imunological markers of elastin degradation in both groups and to assess their relationship with the risk factors characterizing the metabolic syndrome.

Results: When comparing the mean value of AEAb IgG in the control group and subject group with metabolic syndrome (respectively $0,45 /-0.11$ and $0.54 /-0.29$ ) statistically significant higher mean value of AEAb IgG in the group with metabolic syndrome, $t=-1,85, p=0.03$ is observed. When comparing the mean value of ATEAb IgG in the control group and subject group with metabolic syndrome (respectively $0,45 /-0.13$ and $0.55 /-.43$ ) statistically significant higher mean value of ATAb IgG in the group with metabolic syndrome, $F=6,83, p=0.01$ is observed. There isn't a statistically significant difference in AEAb IGM and ATropoEAb IgM in both groups. In the whole sample AEAb IgG showed positive correlation with total cholesterol with a correlation Spearman coefficient $r=$ 0,25 , and $\mathrm{p}=0,02$, with triglyceride levels with Pearson correlation coefficient of $r=0,35, p=0,001$ and with LDL levels with Spearman correlation coefficient $r=0,29$, and $p$ $=0,006$. In the whole sample ATropoEAb IgG showed positive correlation with LDL levels with Spearman correlation coefficient $r=0,29, p=0,006$ and with levels of total cholesterol with a Pearson correlation coefficient $r=$ 0,33 , and $p=0,001$. The Correlations are described by regression analysis and the relationship is linear.

Conclusion: It is proved that the AEAb IgG and AtropoEAb IgG are significantly elevated in the subjects with metabolic syndrome without manifested cardiovascular complications compared with the control group, whereas no difference in AEAb IgM and ATropoEAb IgM has been observed in the both groups.

Key words: Metabolic syndrome, AEAb IgG, ATEAb $\mathrm{IgG}$, risk factor

\section{BACKGROUND:}

It is known that metabolic syndrome characterized by diabetes, hypertension, dyslipidemia, and central obesity is associated with the syndrome of early vessels aging, characterized by a change in elasticity of the vessel wall. The early manifestation of the metabolic syndrome in younger people in the modern society, leads to earlier manifestation of the complications of early vessels aging, and the combination of several risk factors is crucial and leads to acceleration of the vessels aging. Elastin is one of the main building blocks of the of the vessel wall. Its main characteristic is its elasticity, allowing the vessel to restore its shape after stretching or shrinking. Loss of elasticity is a key component in the pathogenesis of cardiovascular complications.

Number of studies have shown that the immune system reflects the physiological changes in the elastin metabolism in the vascular wall. In the recent years, using immunological methods, changes in elastin metabolism is examined, as a sign of vascular aging in high-risk subjects with cardiovascular complications.

There isn't sufficient information about the nature of the immune response against tropoelastin and elastin metabolism in patients with metabolic syndrome without cardiovascular complications. The data on the prognostic value in the evaluation process of early vascular aging is scarce. Therefore, the attempt to determine the changes in elastin and tropoelastin metabolism in metabolic syndrome 
is one of the first steps to seek early immunological rather than instrumental criteria to evaluate changes of the vascular wall.

\section{MATERIALS AND METHODS:}

A study is conducted on 62 subjects with metabolic syndrome without vascular complications and 42 controls.

The main objective of the study was to compare the imunological markers of elastin degradation in both groups and to assess their relationship with the risk factors characterizing the metabolic syndrome.

\section{RESULTS:}

When comparing the average AEAb IgG in the control group and subjects with metabolic syndrome (respectively $0.45+/-0.11$ and $0.54+/-0.29$ ) was determined statistically significantly higher mean values of AEAb $\operatorname{IgG}$ in the group with metabolic syndrome, $\mathrm{t}=-1,85, \mathrm{p}=0.03$, Figure.1.

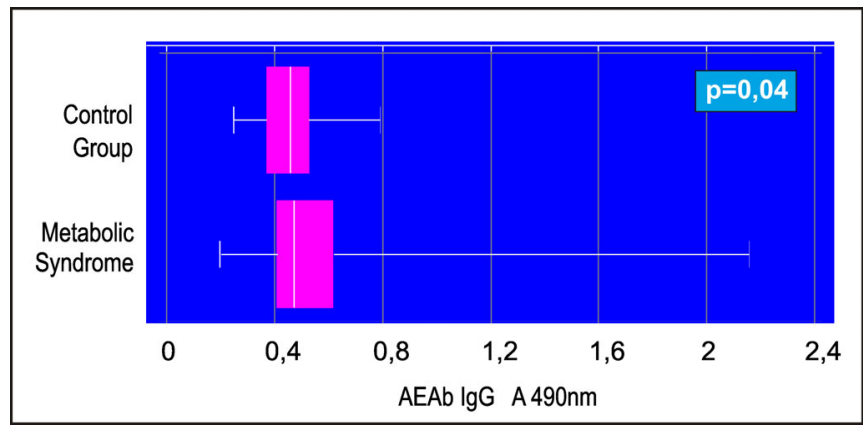

Fig.1. Comparison between the mean AEAb IgG value in healthy subjects and those with metabolic syndrome

The distribution of AEAb IgM in the group of controls and subjects with metabolic syndrome is different from normal. When comparing the medians of the two groups (0.34 and 0.32 , respectively) no statistically significant difference (Mann-Whitney (Wilcoxon) W test $=729.5, \mathrm{p}=$ 0.06)

When comparing the average ATEAb IgG in the group of controls and subjects with metabolic syndrome (respectively $0.45+/-0.13$ and $0.55+/-0.43$ ) was statistically significantly higher values ATEAb IgG in the group with metabolic syndrome, $t=-2.638, p=0.005$, Fig. 2

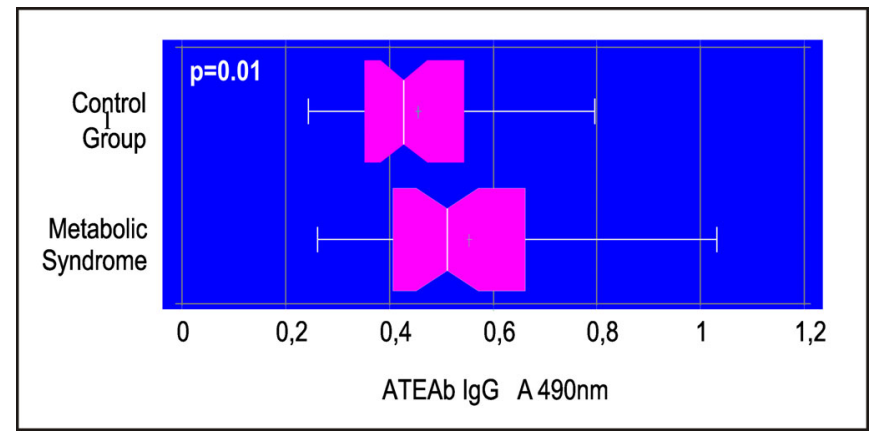

Fig.2. Comparison between the mean ATEAb IgG value in healthy subjects and those with metabolic syndrome

No significant difference was found between the median IgM ATEAb value in healthy subjects and those with metabolic syndrome, respectively 0.28 and $0,25, p>0.05$.

Weak correlation was determined between the AEAb IgG levels in the total group and total cholesterol with a correlation coefficient of Spearman $c=0,25, p=0,02$. Regression analysis best describes this dependence by a linear model, $\mathrm{c}=0,25, \mathrm{p}=0,02$, Fig.3.

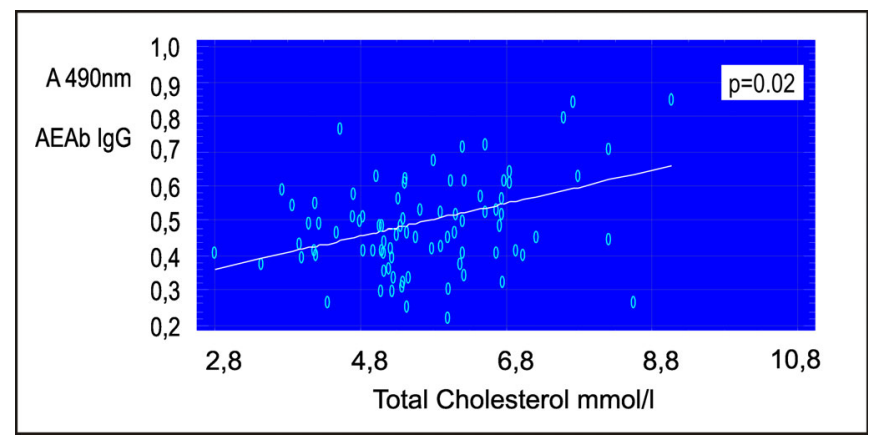

Fig.3. Correlation analysis between AEAb IgG value and total cholesterol in the total group

When evaluating the correlation between AEAb IgG levels in the whole group and triglyceride levels, a moderate correlation was found with a Pearson correlation coefficient $r=0,35$, and $p=0,001$. Regression analysis best describes this relationship by a linear model, $r=0,35, p=0,001$, Fig.4. 


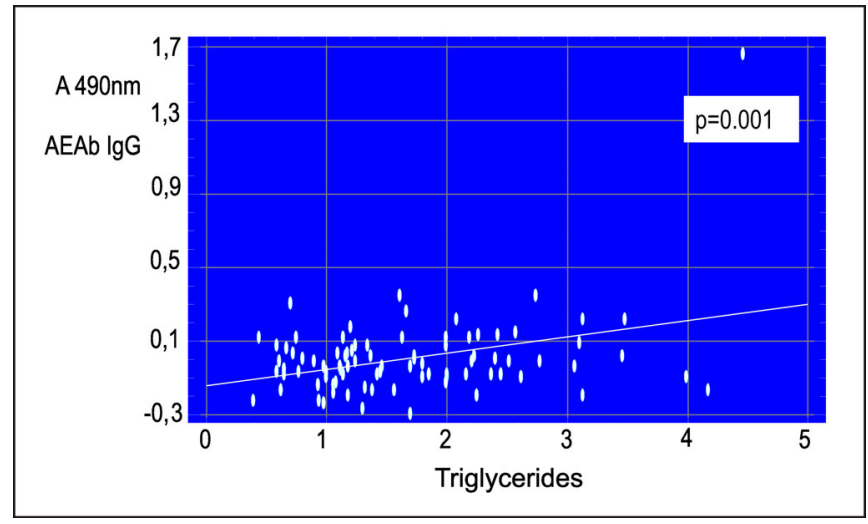

Fig.4. Correlation analisys between AEAb IgG levels and triglycerides in the whole group

In addition to total cholesterol and triglycerides the AEAb IgG levels show weak positive correlation with the LDL levels, Spearman correlation coefficient $c=0,29$, and $p$ $=0,006$. Regression analysis best describes this relationship by a linear model, $\mathrm{c}=0,29, \mathrm{p}=0,006$, fig. 5

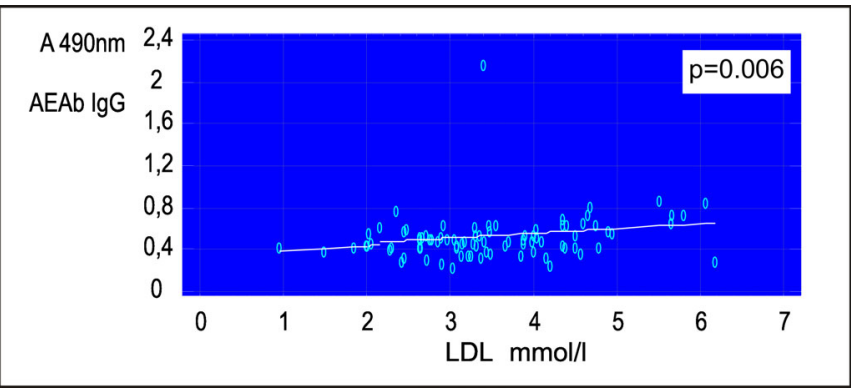

Fig.5. Correlation analysis between AEAb IgG and the levels of LDL in the whole group

Weak but statistically significant positive correlation was determined between the levels of a ATEAb IgG in the whole group with total cholesterol levels with Pearson's $r=$ 0,25 , and $p=0,02$. Regression analysis best describes this relationship which is linear, $r=0,25, p=0,02$, fig.6.

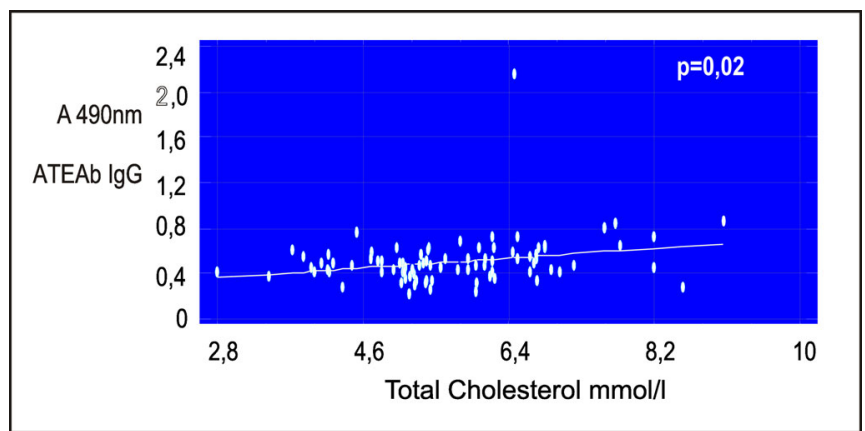

Fig.6. Correlation analysis between ATEAb IgG and total cholesterol levels in the whole group
Statistically significant, moderate, positive correlation was determined when assessing the correlation between ATEAb IgG and total cholesterol levels in the general group with Pearson correlation coefficient $r=0,33$, and $p=0,001$. Regression analysis best describes this relationship which is linear, $r=0,33, p=0,001$, fig. 7

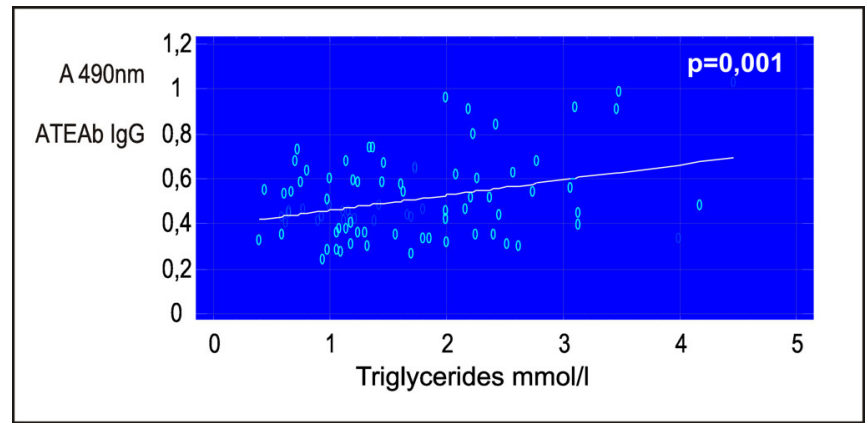

Fig.7. Correlation analysis between IgG ATEAb and triglyceride levels in the total group

\section{CONCLUSION:}

The changes in the immunological markers against elastin and tropoelastin, showed that antibodies from class $\mathrm{IgG}$, characterising the secondary immune response, are elevated in the long-term chronic inflammation characterising the metabolic syndrome and atherosclerosis, respectively, the vascular aging process. Therefore, the constellation of elevated AEAb IgG, ATEAb IgG, can be used as an early marker of vascular aging.

The following conclusion can be made: dyslipidemia and its inherent hypertriglyceridemia is one of the laboratory criteria for metabolic syndrome, Characterising the increased insulin resistancy. Increased insulin resistancy affects the turnover of elastin and tropoelastin. The correlation between elevated levels of AEAb IgG, ATEAb IgG, and triglycerides adequately reflects the process of vascular aging, apparently orchestrated by insulin resistance and therefore can be discussed as a constellation, which is a sure sign of early vascular aging. 


\section{REFERENCES:}

1. Frohlich ED, Susic D. Blood pressure, large arteries and atherosclerosis. In: Safar ME, Frohlich ED (eds): Atherosclerosis, Large Arteries and Cardiovascular Risk. $A d v$ Cardiol. Basel, Karger. 2007; 44:117124. [PubMed] [CrossRef]

2. Vague J. Sexual differentiation, a factor affecting the forms of obesity. Presse Med. 1947; 30:339-40

3. International Diabetes Federation. The IDF worldwide definition of the metabolic syndrome. April 14, 2005 www.idf.org/webdata/docs/ Metac_syndrome_def.pdf

4. Isomaa B, Almgren P, Tuomi T, Forsŭn B, Lahti K, Nissŭn $\mathrm{M}$, et al. Cardiovascular morbidity and mortality associated with the metabolic syndrome. Diabetes Care. 2001 Apr; 24(4):683-689. [PubMed] [CrossRef]

5. Klein BE, Klein R, Lee KE. Components of the metabolic syndrome and risk of cardiovascular disease and diabetes in Beaver Dam. Diabetes Care. 2002 Oct;25(10):1790-4. [PubMed] [CrossRef]

6. Akhtar S, Meek KM, James V. Immunolocalization of elastin, collagen type I and type III, fibronectin, amd vitronectin in extra-cellular matrix components of normal and myxomatous mitral heart valve chordae tendineae. Cardiovasc Pathol. 1999 Jul-Aug;8(4): 203-1. [PubMed] [CrossRef]

7. Mayne R, Brewton RG. New members of the collagen superfamily. Curr Opin Cell Biol. 1993; Oct; 5(5): 883-90. [PubMed]

8. Mecham RP, Hinek A, Grifin GL, Senior RM, Liotta LA. The Elastin receptor shows structural and functional similarities to the $67-\mathrm{kDa}$ tumor cell laminin receptor. $J$ Biol Chem. 1989 Oct 5;264(28):16652-7. [PubMed]

9. Belz GG. Elastic properties and Windkessel function of the human aorta. Cardiovasc Drugs Ther. 1995 Feb;9(1):73-83. [PubMed]

10. Nicoloff G, Weiss AS, Iotova V, Tzaneva V, Petrova C, Domuschieva N, et al. Abnormal levels of serum antielastin antibodies in children with diabetes mellitus type 1. J Investig Med. 2006 Dec;54(8):461-7. [Pubmed] [CrossRef]

11. S. Tisheva. Changes in elastin degradation immunological indices in patients with moderate arterial hypertension maintained on systematic antihypertension treatment. Biotechnol \& Biotechnol Eq. 2005 Sep;19(3):149159.

12. Bizbiz L, Alpŭrovitch A, Robert L. Aging of the vascular wall: serum concentration of elastin peptides and elastase inhibitors in relation to cardiovascular risk factors. The EVA study. Atherosclerosis. 1997 May; 131(1):73-8. [PubMed]

13. Baydanoff S, Nicoloff G, Alexiev C. Age-related changes in the level of circulating elastin-derived peptides in serum from normal and atherosclerotic subjects Atherosclerosis. $1987 \mathrm{Jul} ; 66$ (1-2):163-168. [PubMed]

14. Daskalova M, Taskov H, Dimitrova E, Baydanoff S. Humoral and Cellular Immune Response to Elastin in Patients with Systemic Sclerosis. Autoimmunity. 1997; 25(4):233-241. [PubMed]

15. Nicoloff G, Christova P. Elastin degradation products among obese children with family history of arterial hypertension. Diabetologia Croatica. 2003; 32(1):25-27.

16. Gminski J, Drozdz M, UlfigMaslanka R, Najda J. Evaluation of elastin metabolism in children from families with high risk of atherosclerosis. Atherosclerosis. 1991 Dec;91(3):185-189. [PubMed]

17. Lindholt JS, Heickendorff L, Antonen S, Fasting H, Hennenberg EW. Natural history of abdominal aortic aneurysm with and without coexisting chronic obstructive pulmonary disease. $J$ Vasc Surg. 1998; 28:226-233. [PubMed]

18. Nicoloff G, Baydanoff S, Stanimirova N, Petrova C, Christova P. An association of anti-elastin IgA antibodies with development of retinopathy in diabetic children. Gen Pharmacology. 2000 Aug;35(2):83-87. [PubMed] [CrossRef]

19. Atanasova M, Konova K, Georgieva M, Dimitrova A, CoquandGandit M, Faury G, et al. Age-Related Changes of Anti-Elastin Antibodies in Senescence-Accelerated Mice. Gerontology. 2010; 56(3):310-318. [PubMed] [CrossRef]

20. Fulop T Jr, Jacob MP, Robert L. Determination of anti-elastin peptide antibodies in normal and arteriosclerotic human sera by ELISA. J Clin Lab Immunol. 1989 Oct;30(2):69-74. [PubMed]

21. Bako G, Jacob MP, Fulop T Jr, Foris G, Leovey A, Robert L. Immunology of elastin: study of anti-elastin peptide antibodies by DOT immunobinding assay. Immunol Lett. 1987 Jul;15(3):187-192. [PubMed]

\section{Address for correspondence:}

Dr. Edward Mekenyan

First Cardiological Clinic, UMHAT “G. Stranski” - Pleven

8A, Georgi Kochev str., Pleven, Bulgaria

Phone: 0035964886140

Email: edward_mekenyan@yahoo.com; 